\title{
OPTIMAL DESIGN AND NUMERICAL SIMULATION ON FISH-LIKE FLEXIBLE HYDROFOIL PROPELLER
}

\author{
Gang Xue ${ }^{1)}$, Ph. D, \\ Yanjun Liu' ${ }^{1)}$, Prof., PH. D, \\ Muqun Zhang'), Master \\ Wei Zhang'), Ph. D, \\ Jian Zhang'), Ph. D, \\ Huaqing Luo ${ }^{1)}$, Master \\ Rui Jia ${ }^{1)}$, Master \\ 1) Key Laboratory of High Efficiency and Clean Mechanical Manufacture, Ministry of Education, \\ School of Mechanical Engineering, Shandong University, Ji'nan, China
}

\begin{abstract}
Hydrofoil is widely used in underwater vehicle for the excellent hydrodynamic characteristics. Currently, researches are mostly about the rigid hydrofoil while the flexible hydrofoil, like the caudal fin, has not been studied adequately. In this paper, the fish was regarded as the bionic object. Then the kinematics model to describe the fish swimming was put forward. A fin-peduncle propulsion mechanism was designed based on the kinematics model to achieve the similar sine curve swimming model. The propulsion mechanism was optimized by Matlab to reduc the deviation between the output curve of the fin-peduncle propulsion mechanism and the ideal motion trajectory. Moreover, the motion phase angles among flexible articulations are optimized to reduce fluid resistance and improve propulsive efficiency. Finally, the fish-like hydrofoil oscillation is simulated by fluid-solid coupling method based on the Fluent. It was shown that the optimized flexible fish-like oscillation could generate the motion that follows the similar law of sine. The propulsive efficiency of oscillating hydrofoil propeller is much higher than that of the screw propeller, and the flexible oscillation has higher propulsive efficiency than the rigid oscillation without obvious fluid resistance increase.
\end{abstract}

Keywords: -bionics; flexible hydrofoil; phase angle; hydrodynamic coefficient

\section{INTRODUCTION}

Autonomous Underwater Vehicle (AUV) is an important device for marine operations, which has been used as an important tool for submarine pipeline laying, subsea equipment maintenance, etc. The marine organisms have benefited the natural evolution for millions of years. It will do a lot favor to introduce bionics into the study of AUV. Fish could swim in high speed and good maneuverability under water. It is observed that the dolphin could swim in 3 times to 7 times of the body length per second and the turning radius can be $23 \%$ to $30 \%$ of the body length [1]. In 1994, MIT developed the world's first fish-like robot, Robo Tuna, whose maximum speed was $2 \mathrm{~m} / \mathrm{s}$ [2]. After that, more attentions have been attracted on the fish-like robot. The PF-300 with two motors controlling the fish-like body collaboratively was designed by Japanese researchers [3]. Harbin Engineering
University developed a fish-like robot to imitate the atlantic bluefin tuna with a three-link tail [4]. Du Ruxu developed a cable-driven robot fish, whose body was flexible and the propulsion efficiency could reach 65\% [5]. Beihang University developed a series of fish-like robots, SPC, which can swim as long as $70.2 \mathrm{~km} \mathrm{[6].}$

Besides, a number of numerical studies on the hydrodynamic characteristics of oscillating hydrofoil have been performed. The slender-body theory was put forward for the deformable body to describe the conditions in which fish will benefit higher Froude propulsion efficiency [7]. The numerical simulation method on the three-dimensional flexible fish model was employed to study the hydrodynamic characteristics of swimming motion [8]. Lagrangian coherent structures (LCS) defined by the ridges of the finite-time Lyapunov exponent (FTLE) was utilized in this study to elucidate the time-dependent turbulent flows around a Clark-Y hydrofoil [9]. 
Compared with the screw propeller, the fish-like flexible oscillating hydrofoil propeller has higher propulsive efficiency and better maneuverability. In this research, the fin-peduncle propulsion mechanism was designed and optimized to simulate the fish swimming. Besides, the phase angles among flexible articulations are optimized to reduce fluid resistance and improve propulsive efficiency. Finally, the fishlike hydrofoil oscillation is simulated by fluid-solid coupling method based on the Fluent.

\section{PROPULSION MECHANISM}

\section{KINEMATICS MODEL}

According to the observation of fish swimming, an equation to describe the centerline trajectory of the swimming fish body was put forward [10].

$$
h\left(x_{n}, t\right)=H\left(0.21-0.66 x_{n}+1.1 x_{n}^{2}+0.35 x_{n}^{8}\right) \sin (2 \pi f t)
$$

where $x_{n}$ is the ratio of the body coordinate value (marked as $x$ ) and the body length (marked as $L$ ), $t$ is the movement time, $h\left(x_{n}, t\right)$ is the oscillation amplitude of the body, $H$ is the maximum oscillation amplitude at the end of the caudal peduncle, $f$ is the oscillating frequency of the caudal peduncle.

After studying the oscillating movements of the fish body, Wang simplified the kinematics model [11]. Supposing that the maximum oscillation amplitude of the fish head is one tenth of the body length and the oscillation amplitude of the barycenter is zero, the simplified equation for the centerline trajectory of the fish body can be described as:

$$
h\left(x_{n}, t\right)=H\left(0.1-1.3 x_{n}+2.2 x_{n}^{2}\right) \sin (2 \pi f t)
$$

The centerline trajectories of the fish body at different moment are shown in Fig. 1.

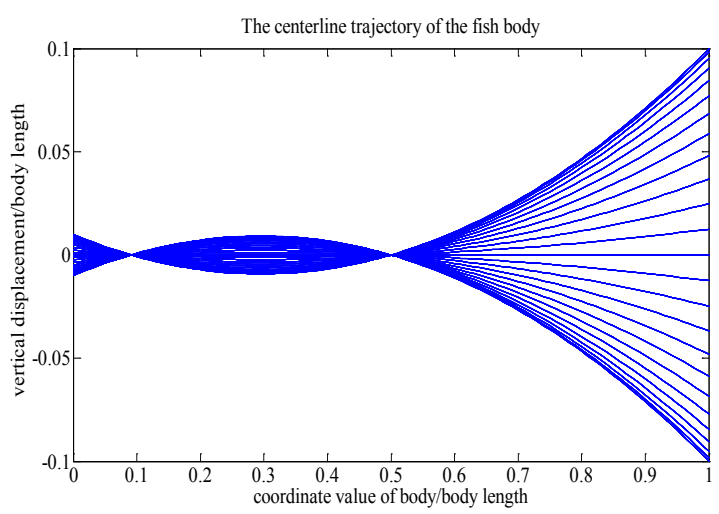

Fig. 1 The centerline trajectories of the fish body

The kinematics equation at the end of the tail can be described as:

$$
h(t)=H \sin (2 \pi f t)
$$

The pitching movement of the caudal fin lags behind its plunging movement, which can be described as:

$$
\theta(t)=\theta_{\max } \sin (2 \pi f t+\varphi)
$$

where $\theta_{\max }$ is the maximum oscillation angle, and $\varphi$ is the phase angle between the plunging movement and the pitching movement.

The swimming speed of the fish can be marked as $\mathrm{v}$ and the length of the caudal fin can be marked as 1 . The kinetic coordinate system for caudal fin can be established, as shown in Fig. 2.

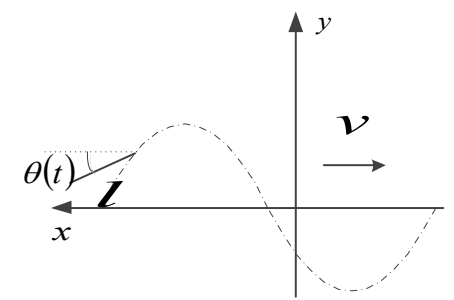

Fig. 2 The kinetic coordinate system for caudal fin

In Fig. 2, the full line is the caudal fin and the dotted line is the plunging movement trajectory of the caudal fin.

The coordinate value for the movement of the caudal fin at any time in the kinetic coordinate can be described as:

$$
\begin{gathered}
x=v t-l \cos \left[\theta_{\text {max }} \sin (2 \pi f t+\varphi)\right] \\
y=H \sin (2 \pi f t)-l \sin \left[\theta_{\text {max }} \sin (2 \pi f t+\varphi)\right.
\end{gathered}
$$

\section{MECHANISM DESIGN}

The propulsion mechanism for the fish-like flexible oscillating hydrofoil propeller was designed based on the kinematics model. The mechanism sketch is shown in Fig. 3.

As shown in Fig. 3, member AB, rotating around A, is riven by the servo motor. The rotation is transformed into the sinusoidal reciprocating motion of member $\mathrm{CD}$, which is connected with member $\mathrm{AB}$ by prismatic joint and revolute joint. Member CD and member EF are connected to member DE by revolute joints. Member EF is fixed to member FG. Member CD will drive member DE, member EF, and member FG to achieve the sinusoidal movement output. An actuator is fixed at the end of member FG to control the caudal fin swinging lagged behind member FG in certain phase angle independently. 


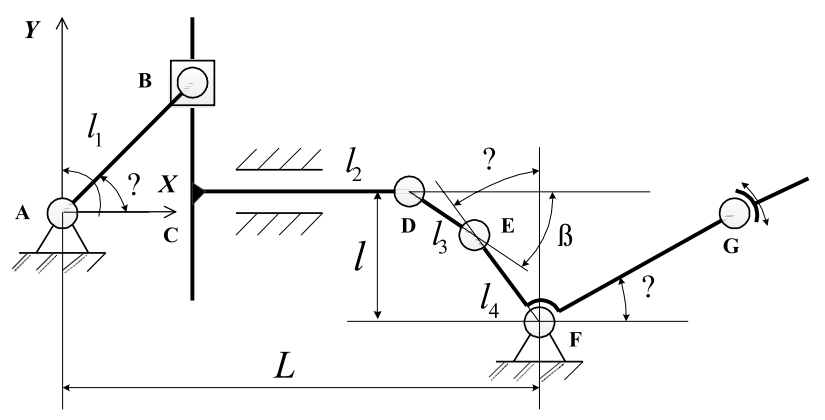

Fig. 3 The mechanism sketch of the propulsion mechanism

In Fig. 3, a coordinate system is established where the origin is point $\mathrm{A}$. Point $\mathrm{F}$ is fixed on the coordinate. As the position relations shown in Fig. 3, some formulas can be deduced as:

$$
\begin{gathered}
l_{3} \sin \beta+l_{4} \cos \alpha=l \\
x_{C}=l_{1} \cos \theta \\
x_{D}=l_{1} \cos \theta+l_{2} \\
x_{E}=l_{1} \cos \theta+l_{2}+l_{3} \cos \beta \\
x_{F}=l_{1} \cos \theta+l_{2}+l_{3} \cos \beta+l_{4} \sin \alpha \\
l_{1} \cos \theta+l_{2}+\sqrt{l_{3}^{2}-\left(l-l_{4} \cos \alpha\right)^{2}}+l_{4} \sin \alpha=L
\end{gathered}
$$

where $x_{C}, x_{D}, x_{E}$, and $x_{F}$ are the coordinate values of $\mathrm{C}$, $\mathrm{D}, \mathrm{E}$, and F. $l_{1}, l_{2}, l_{3}$, and $l_{4}$ are the length of member $\mathrm{AB}$, member $\mathrm{CD}$, member DE, and member EF. $l$ is the distance between point $\mathrm{D}$ and point $\mathrm{F}$ in $\mathrm{Y}$ direction. $L$ is the distance between point $\mathrm{A}$ and point $\mathrm{F}$ in $\mathrm{X}$ direction. $\alpha, \beta$, and $\theta$ are the angles as shown in Fig. 3.

The propeller model was designed based on the mechanism sketch, as shown in Fig. 4.

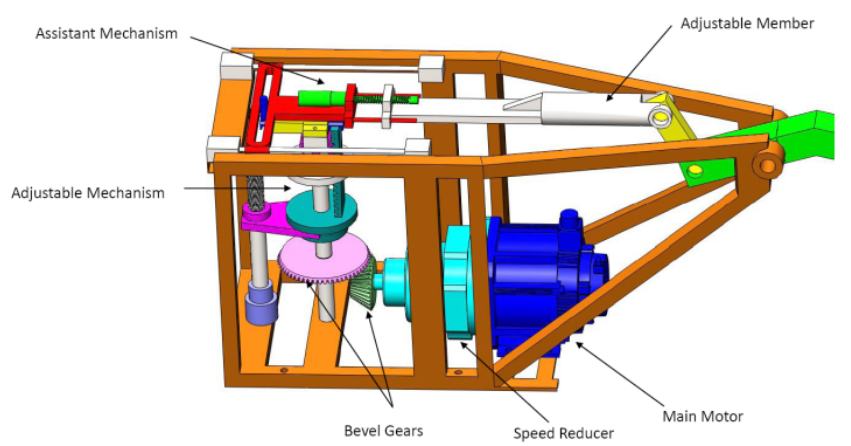

Fig. 4 The propeller model
The length of the adjustable member can be changed. If the adjustable member is shortened, the balanced position for the plunging movement of caudal peduncle will move up, and if the adjustable member is lengthened, it will move down. So the swimming direction of the fish-like robot will be controlled by the adjustment of the adjustable member length.

\section{OPTIMIZATION BASED ON MATLAB}

The length of the mechanism members affect the plunging movement of caudal peduncle, which increases the difficulty of the propulsion mechanism design. At present, the most used method for the mechanism design with the given motion law or the motion trajectory is the function approximation method [12]. However, it is very complicated for the multivariable mechanism optimization. Matlab contains a variety of optimization toolbox, which can be applied in the design optimization of the mechanism to improve the efficiency and accuracy of the calculation.

The propulsion mechanism can be simplified as Fig. 5 shown.

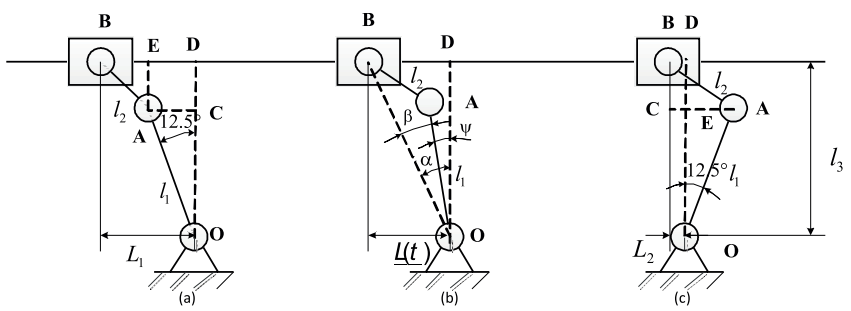

Fig. 5 Mechanism Moment Position

The left limit position is shown in position (a), the right limit position is shown in position (c), and position (b) shows any moment position of the movement. The block $\mathrm{B}$ goes the sinusoidal reciprocating motion.

The oscillating frequency of the plunging movement is about $2.1 \mathrm{~Hz}$ and theW range of that is about $25^{\circ}$ according to the observation. It means the oscillating range of member $\mathrm{OA}$ is about $12.5^{\circ}$. The length of member OA (marked as $l_{1}$ ), the length of member $\mathrm{AB}$ (marked as $l_{2}$ ), the vertical height between point $\mathrm{B}$ and point $\mathrm{O}$ (marked as $l_{3}$ ), and the movement stroke of block B (marked as $L$ ) are the main parameters to affect the final output of the mechanism. $l_{1}$, $l_{2}$, and $l_{3}$ are the design variables and the follow equation can be established as:

$$
x=\left[l_{1} ; l_{2} ; l_{3}\right]^{T}
$$

As shown in Fig. 5(b), the horizontal distance between point $\mathrm{B}$ and point $\mathrm{O}$ can be described by $L(t)$, as:

$$
L(t)=0.5 L \cos 4 \pi t+\frac{L_{1}+L_{2}}{2}
$$

where $t$ is the movement time, $L_{1}$ and $L_{2}$ are the 
horizontal distance of the left limit position and the right position between point $\mathrm{B}$ and point $\mathrm{O}$.

As shown in Fig. 5(a) and Fig. 5 (c), $L_{1}$ and $L_{2}$ can be described as:

$$
\begin{aligned}
& L_{1}=\sqrt{l_{2}^{2}-\left(l_{3}-l_{1} \cos 12.5^{\circ}\right)^{2}}+l_{1} \sin 12.5^{\circ} \\
& L_{2}=\sqrt{l_{2}^{2}-\left(l_{3}-l_{1} \cos 12.5^{\circ}\right)^{2}}-l_{1} \sin 12.5^{\circ}
\end{aligned}
$$

The ideal oscillating law of member OA can be described by $\Psi$, as:

$$
\Psi=12.5^{\circ} \sin 4 \pi t
$$

To minimize the square of the difference between the mechanism output angle and the ideal output angle, the target function can be described as:

$$
F(x)=\sum_{t=0}^{0.25}[\Psi(x)-\Psi]^{2}
$$

where $\Psi(x)$ is the oscillating angle of member OA, which can be described as:

$$
\Psi(x)=\alpha(t)-\beta(t)
$$

where $\alpha(t)$ and $\beta(t)$ can be calculated from $\triangle \mathrm{OBD}$ and $\triangle \mathrm{OAB}$ as:

$$
\begin{gathered}
\alpha(t)=\arccos \left[\frac{l_{3}}{\sqrt{l_{3}^{2}+L^{2}(t)}}\right] \\
\beta(t)=\arccos \left\{\frac{l_{1}^{2}+\left[l_{3}^{2}+L^{2}(t)\right]-l_{2}^{2}}{2 l_{1} \sqrt{l_{3}^{2}+L^{2}(t)}}\right\}
\end{gathered}
$$

The swimming resistance is little with the streamlined shape. It is shown that the swimming resistance will reduce $75 \%$ with the slender ratio of the fish body being 4.5 under the bionics theory. In this research, the length of the fish-like robot body was set as $0.9 \mathrm{~m}$, so the widest of the body is no more than $0.2 \mathrm{~m}$. Besides, in order to make the robot swim autonomously, the swimming parameters should be variable according to different conditions, so the movement stroke of block B cannot be too small.

To achieve the good transmission of the mechanism, the minimum transmission angle should be larger than $40^{\circ}$. The two limit positions of the movement are shown in Fig. 6.The transmission angle at the limit position should be larger than $40^{\circ}$, which will make sure the good transmission at any time of the movement.

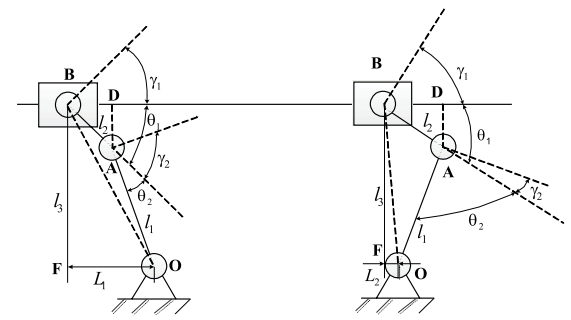

Fig. 6 The transmission angle at the limit position $\theta_{1}$

In Fig. 6, the constraint conditions of the pressure angle at the limit position can be obtained from $\triangle \mathrm{ADB}$, as:

$$
\frac{l_{3}-l_{1} \cos 12.5^{\circ}}{l_{2}} \leq \sin 50^{\circ}
$$

In Fig. 6, the constraint conditions of the pressure angle $\theta_{2}$ at the limit position can be obtained from $\triangle \mathrm{OAB}$, as:

$$
\begin{aligned}
& \frac{L_{1}^{2}+l_{3}^{2}-l_{1}^{2}-l_{2}^{2}}{2 l_{1} l_{2}} \geq \cos 50^{\circ} \\
& \frac{L_{2}^{2}+l_{3}^{2}-l_{1}^{2}-l_{2}^{2}}{2 l_{1} l_{2}} \geq \cos 50^{\circ}
\end{aligned}
$$

The optimization of rod mechanism is simple nonlinear programming problem, and the fmincon function in Matlab toolbox is available. Therefore, the target function was calculated every $0.02 \mathrm{~s}$ and all the results were accumulated to a sum. According to the optimization, the optimal design variables of the solution are $x=[143.605 ; 62.196 ; 187.8459]^{T}$ , which means $l_{1}=143.605 \mathrm{~mm}, l_{2}=62.196 \mathrm{~mm}$, and $l_{3}=187.8459 \mathrm{~mm}$. The lengths are set as $l_{1}=144 \mathrm{~mm}$ , $l 62 \mathrm{~mm}$, and $l_{3}=188 \mathrm{~mm}$ for manufacturing and the output angle of the mechanism is compared with the ideal output angle, as shown in Fig. 7.

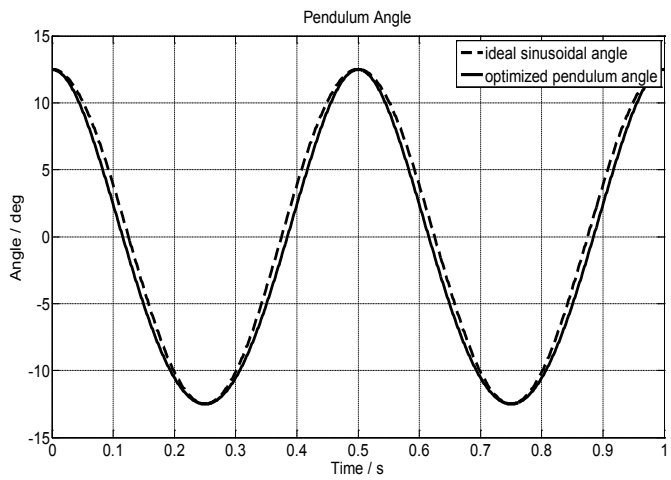

Fig. 7 The output angle of the mechanism and the ideal output angle

In Fig. 7 , the dotted line is the ideal outputs angle and the solid line is the actual output angle of the designed mechanism. It comes to the conclusion that the actual output angle amplitude of the designed propulsion mechanism is $12.5^{\circ}$ and changes follow the similar law of sine, which is similar to the ideal output angle. 


\section{STUDY OF PHASE ANGLE}

The forces on the caudal fin were analyzed, as shown in Figure 8.

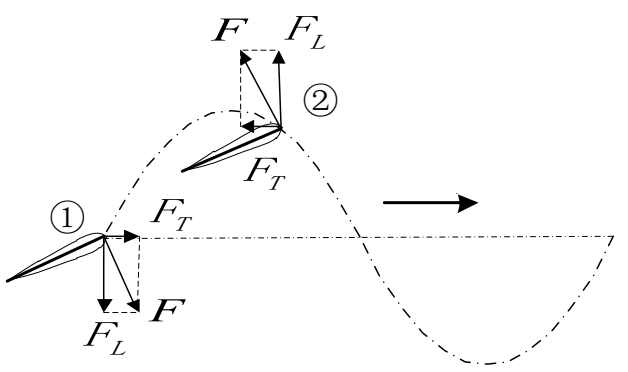

Fig. 8 Analysis of the forces on caudal fin

In Fig. $8, F_{T}$ is the thrust force, which goes along the forward direction at position (1), and it is positive. The caudal fin moves down with the caudal peduncle at position (2), and the force direction is perpendicular to the caudal fin upward. The thrust force goes along the reverse-forward direction, which is negative.

As the observation of the fish swimming, the relation between the swimming velocity and the oscillating frequency is linear. Besides, the oscillation amplitude of the caudal peduncle is essentially unchanged during the swimming process. The biologists point out that the amplitude of the caudal peduncle oscillation is about 0.17 to 0.25 times of the body length. Therefore, the movement parameters of the fish-like robot are as follows:

Swimming speed: $v=L \mathrm{~m} / \mathrm{s}$;

Maximum oscillation range of the caudal fin: $H=0.1 \mathrm{~L} \mathrm{~m}$;

Frequency of the oscillation: $f=2 \mathrm{~Hz}$;

Maximum oscillation angel range of the caudal fin: $\theta_{\max }=30^{\circ}$.

The phase differences between the pitching movement of caudal fin and the plunging movement of caudal peduncle are set as $60^{\circ}, 90^{\circ}$ and $120^{\circ}$ for the kinematics simulation. The oscillation of the caudal fin and the motion trajectory of the swing shaft are shown in Fig. 9 to Fig. 11

As the phase difference is $60^{\circ}$, the caudal fin produces more negative force than positive negative force, and it is the same as the phase difference is $120^{\circ}$. When the phase difference is $90^{\circ}$, the caudal fin produces positive thrust all the period. So it is considered that the phase difference between the caudal fin plunging movement and pitching movement controls the attack angle of the caudal fin movement, and $90^{\circ}$ is the best phase angel.

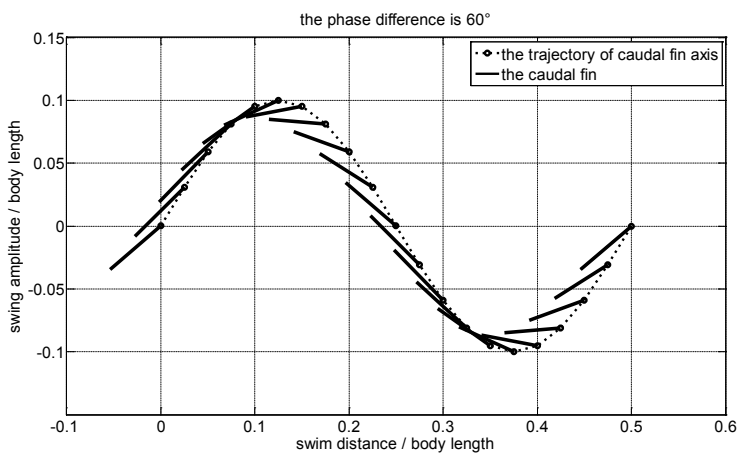

Fig. 9 The phase difference is $60^{\circ}$

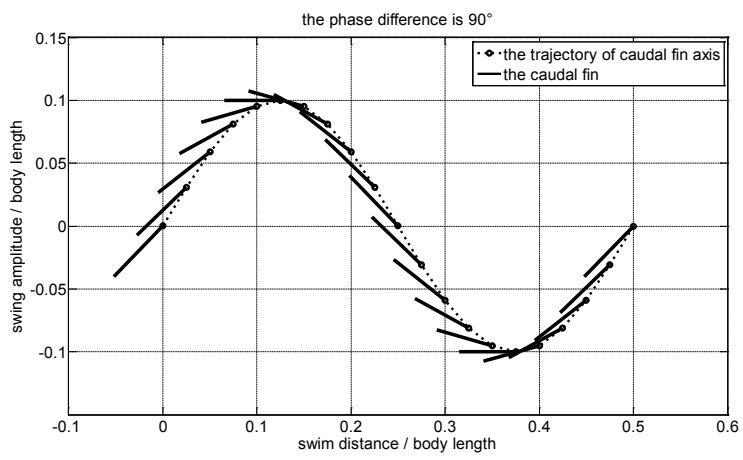

Fig. 10 The phase difference is $90^{\circ}$

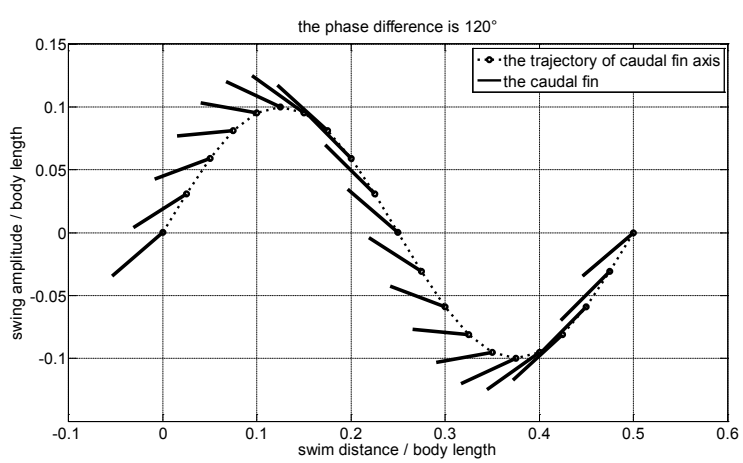

Fig. 11 The phase difference is $135^{\circ}$

\section{HYDRODYNAMIC COEFFICIENTS ANALYSIS}

The hydrodynamic coefficients can be defined as:

$$
\begin{gathered}
c_{d}=\frac{F_{d}}{\frac{1}{2} \rho C_{0} v^{2}} \\
c_{l}=\frac{F_{l}}{\frac{1}{2} \rho C_{0} v^{2}} \\
c_{m}=\frac{M}{\frac{1}{2} \rho C_{0}^{2} v^{2}}
\end{gathered}
$$


where $c_{d}$ is drag coefficient, $c_{l}$ is lateral force coefficient, $c_{m}$ is moment coefficient, $F_{d}$ is drag produced by flexible oscillating hydrofoil, $F_{l}$ is lateral force produced by flexible oscillating hydrofoil, $M$ is oscillation moment around the head of the fish body produced by flexible oscillating hydrofoil, $\rho$ is fluid density, $C_{0}$ is chord length of the hydrofoil, $v$ is flow velocity of the fluid.

The SIMPLE Method (Semi-implicit Method for PressureLinked Equations) was used to solve the pressure-velocity coupling equations, and the UDF (User Defined Function) was used to achieve the flexible oscillation in the commercial software Fluent. In the simulation, the oscillation frequency of the fish body and the caudal fin is $2 \mathrm{~Hz}$, the hydrofoil is the airfoil of NACA0014, the chord length of fish body is $200 \mathrm{~mm}$, the chord length of caudal fin is $60 \mathrm{~mm}$, and the oscillating scope of caudal fin is $\pm 45^{\circ}$.

In the kinematics model, $H$ is the maximum lateral oscillation amplitude at the end of the fish body, which could express different flexibilities in different values. The flexibility is better with larger lateral oscillation amplitude. If $H$ is zero, the fish body is rigid without any oscillation, but the caudal fin will oscillate as usual.

The hydrodynamic characteristics of flexible oscillating hydrofoil were analyzed in the still water under the condition that the fish oscillated in situ. To use the commercial software Fluent, a minor water flow velocity should be available. The flow velocity was set as $0.01 \mathrm{~m} / \mathrm{s}$ to avoid distinct influence on the flow field.

The curves of the drag coefficient and the lateral force coefficient of fish body in a period in the still water with different oscillation amplitude are shown in Fig. 12.
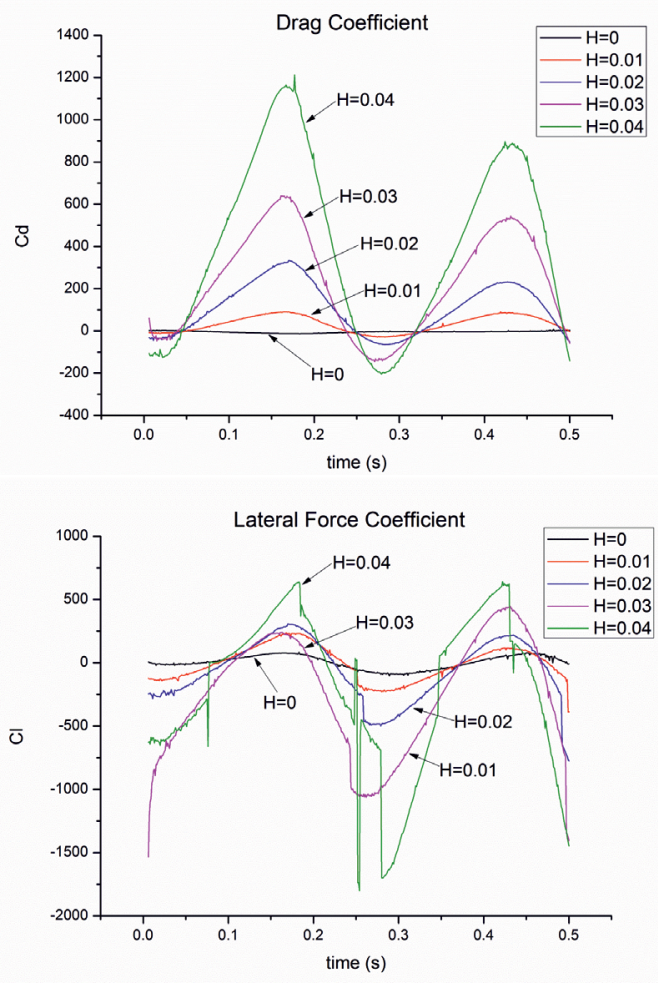

Fig. 12 Drag coefficient curves and lateral force coefficient curves of fish body in the still water
The drag coefficient curves indicate that the drag coefficient approximates a straight line when the fish body doesn't oscillate $(H=0)$, which means the fish body produce no thrust force. The amplitude of the drag coefficient increases with the flexible oscillating amplitude of the fish body getting larger. There are two peak values in a fish body oscillation period and the drag coefficient variation period is half of the fish body oscillation period. The maximum values appear at the $1 / 4$ period and $3 / 4$ period of the fish body oscillation, which means the fish body produces the maximum thrust at these two moments. The integration value during the whole period is positive, which indicates that the fish could go forward with the fish body oscillation in the still water.

As shown in the lateral force coefficient curves, the amplitude and fluctuation of the lateral force increase with the flexibility getting better, and the lateral force generated by the oscillation is unstable. At the $1 / 2$ period of the oscillation, the immense change occurs because the fish body oscillates from one side of the center line to another side. The lateral force consumes the energy generated by the oscillation and increases the swimming instability.

The curves of the drag coefficient and the lateral force coefficient of caudal fin during an oscillation period in the still water with different oscillation amplitude are shown in Fig. 13.
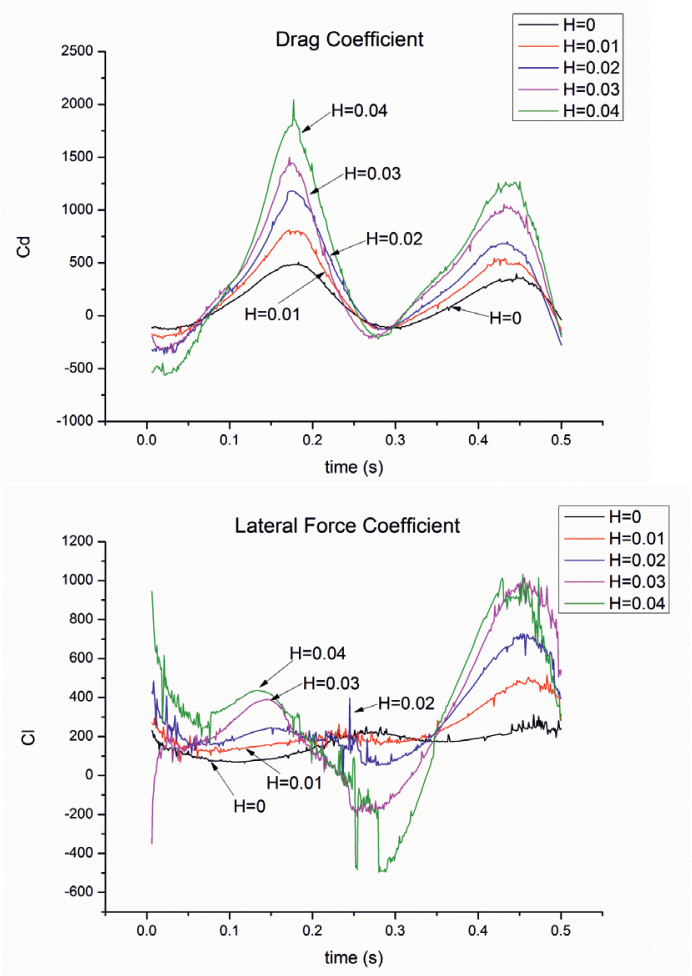

Fig. 13 Drag coefficient curves and lateral force coefficient curves of caudal fin in the still water

As shown in drag coefficient curves, the change trend of the drag coefficient produced by the caudal fin oscillation is same as that of the fish body oscillation. The amplitude of the caudal fin drag coefficient increases with the flexibility and 
the oscillation amplitude of the fish body getting larger. The comparison between the drag coefficient curves of fish body and caudal fin shows that the drag coefficient value of the caudal fin is bigger, which means that the wake field generated by the fish body oscillation promotes the drag coefficient produced by the caudal fin compared with the oscillation without wake field. The drag coefficient values are almost positive in the whole period, which means the oscillation of the caudal fin has evident effects on the propulsion.

As shown in lateral force coefficient curves, the lateral force generated by the caudal fin oscillation increases with the flexibility and oscillation amplitude of the fish body getting larger under the same angle oscillation amplitude of the caudal fin, which indicates that more energy generated by the caudal fin oscillation is consumed. Most of the lateral force coefficient values are positive and the integration during an oscillation period is not equal to zero, which indicates that the caudal fin oscillation would accumulate to unidirectional force and go against the swimming. Accordingly, the unidirectional force should be reduced with the actuator controlling the amplitude and phase of the caudal fin oscillation in the fishlike robot development.

\section{CONCLUSION}

In this research, the kinematics model for the fish-like robot movement was established and the propulsion mechanism was designed based on the model. The length of members in the propulsion mechanism was optimized by the function fimincon in Matlab. The motion curves of the fin-peduncle propulsion mechanism are proven to be similar with that of the ideal motion.

The phase difference between the pitching movement of caudal fin and the plunging movement of caudal peduncle was studied. When the phase angle is $90^{\circ}$, the propulsion mechanism would produce thrust in the whole motion period without any resistance. Meanwhile, the advantage of the articulatory fin-peduncle mechanism is obvious compared with the single oscillating caudal fin.

According to the curves of the hydrodynamic coefficients, the variation period of the drag coefficient and lateral force coefficient is half of the fish body oscillation period, and the variation period of the moment coefficient is as same as the fish body oscillation period. In the still water, the larger the flexible oscillation amplitude is, the bigger the lateral force integration value in a period is, and more energy is consumed.

\section{CONFLICT OF INTEREST}

The authors declared that they have no conflicts of interest to this work.

\section{ACKNOWLEDGEMENTS}

The research was supported by the front and emerging discipline team oriented project of Shandong University "marine resources and utilization of key scientific research" (project number 2014QY006) and "The Fundamental Research Funds of Shandong University” (2016JC035), hereby thanks.

\section{BIBLIOGRAPHY}

1. Nagai, M. (2002). Thinking fluid dynamics with dolphins. US: IOS Press.

2. Triantafyllou, M. S., and Triantafyllou, G. S. (1995). An efficient swimming machine. Scientific American, 272(3): 64-70.

3. Koichi, H., Tadanori, T., and Kenkichi, T., 2000, "Study on turning performance of a fish robot," First International Symposium on Aqua Bio-Mechanisms, pp. 287-292.

4. Cheng, W., 2004, "Research on Simulation and Control Technology for Bionic Underwater Vehicle,” Ph.D. thesis, Harbin Engineering University, Harbin, China.

5. Du, R. X., Li, Z., Kamal, Y. T., Pablo, V. A., 2015, "Robot Fish," New York: Springer Tracts in Mechanical Engineering.

6. Liang, J. H., Zhang, W. F., Wen, L., Wang, T. M., Liu, and Y. J. (2010). Propulsion and Maneuvering Performances of Two-Joint Biorobotic Autonomous Underwater Behicle SPC-III. Robot, 32(6):726-731.

7. Lighthill, M. J. (1960), Note on the swimming of slender fish, Journal of Fluid Mechanics, Vol.9: 305-317.

8. Leroyer, A., Visonneau, M.(2005), Numerical methods for RANSE simulations of a self-propelled fish-like body, Journal of Fluids and Structures, Vol.20, No.7: 975-991.

9. Zhao, Y., Wang G. Y., Huang, B., Wu, Q., Wang, F. F. (2015), Lagrangian-Based Investigation of Unsteady Vertex Structure Near Trailing Edge of a Hydrofoil, Transactions of Beijing Institute of Technology, Vol.35, No.7: 666-670.

10. Romaneko, E. V. (2002). Fish and dolphin swimming. Bulgaria: Pensoft Publishers.

11. Wang, L., Yu, J. Z., Hu, Y. H., Fan, R. F., Huo, J. Y., and Xie, G. M. (2006). Mechanism design and motion control of robotic dolphin. Acta Scientiarum Naturalium Universitatis Pekinensis, 42(3): 294-301.

12. Wang, W. J. (2006). Optimal design of planar linkage based on Matlab optimization toolbox. Light Industry Machinery, 24(4): 76 
CONTACT WITH THE AUTHORS

Yanjun Liu Prof.

e-mail:lyj111ky@163.com

Key Laboratory of High Efficiency and Clean Mechanical Manufacture Ministry of Education School of Mechanical Engineering Shandong University, Ji'nan

\section{CHINA}

